\title{
Changes in Incidence and Survival by Decade of Patients With Primary Colorectal Lymphoma: A SEER Analysis
}

\author{
Qingguo $\mathrm{Li}^{1,2 \dagger}$, Shaobo Mo ${ }^{1,2 \dagger}$, Weixing Dai ${ }^{1,2 \dagger}$, Yaqi $\mathrm{Li}^{1,2}$, Ye $\mathrm{X} \mathrm{u}^{1,2}$, Xinxiang $\mathrm{Li}^{1,2 *}$, \\ Guoxiang Cai ${ }^{1,2 *}$ and Sanjun Cai ${ }^{1,2 *}$
}

${ }^{1}$ Department of Colorectal Surgery, Fudan University Shanghai Cancer Center, Shanghai, China, ${ }^{2}$ Department of Oncology, Shanghai Medical College, Fudan University, Shanghai, China

\section{OPEN ACCESS}

Edited by:

Dora ll'yasova,

Georgia State University,

United States

Reviewed by:

Zhong Jian,

University of California, Davis,

United States

Pier Paolo Piccaluga,

University of Bologna, Italy

*Correspondence:

Xinxiang L

Ixx1149@163.com

Sanjun Cai

caisanjun_sh@163.com

Guoxiang Cai

gxcai2015@163.com

†These authors have contributed equally to this work

Specialty section:

This article was submitted to Epidemiology,

a section of the journal

Frontiers in Public Health

Received: 22 July 2019 Accepted: 11 September 2020 Published: 16 October 2020

Citation:

Li Q, Mo S, Dai W, Li Y, XU Y, Li X, Cai $G$ and Cai $S$ (2020) Changes in Incidence and Survival by Decade of

Patients With Primary Colorectal Lymphoma: A SEER Analysis.

Front. Public Health 8:486401.

doi: 10.3389/fpubh.2020.486401
Purpose: To reveal changes in the incidence, treatment, and survival of patients with colorectal lymphoma.

Methods: Patients diagnosed with primary colorectal lymphoma (PCL) or lymphoma between 1973 and 2014 were identified in the SEER registry. The incidence was estimated by age and join-point analysis. The incidence of different subtypes and the surgical resection rates were compared over different time periods.

Results: The PCL incidence increased from 1.4 per 1000000 people in 1973 to 3.5 in 2014, with an annual percentage change (APC) of 1.98\% (95\% confidence interval [Cl]: $1.29-2.68 \%, P<0.001$ ) from 1985 to 2014 . No statistically significant change was found between 1973 and 1984. For people younger than 60 years, there was a slight increase in PCL incidence, from 0.6 to 1.4\%, from 1973 to 2014. For people age 60 or older, there was a statistically significant increase in PCL incidence from 5.4 to $14.1 \%$ over the same time period. The 5-year cause-specific survival (CSS) for PCL improved markedly from $41.6 \%$ in the period $1973-1976$ to $80.2 \%$ in the period $2009-2012(P<0.001)$. Conversely, the proportion of patients who received surgical therapy decreased gradually from 83.3-100 to 47.7-52.6\% throughout the studied time period.

Conclusions: The incidence of PLC has increased in recent decades. The 5-year CSS of PCL increased continuously, while the rate of surgical resection decreased steadily. These changes in survival trends and therapy strategies indicate that PCL can be well-managed with newer therapeutic reagents.

Keywords: primary colorectal lymphoma, incidence, survival analysis, treatment, SEER

\section{INTRODUCTION}

Lymphoma is the seventh-most common cancer type in the United States and is the seventhleading cause of cancer-related deaths among both men and women. The incidence of lymphoma has been increasing in recent decades $(1,2)$. Approximately $40 \%$ of lymphomas have extranodal manifestations, and the most common site of extranodal involvement is the gastrointestinal tract (3). The incidence of primary colorectal lymphoma (PCL) is rare, accounting for only $0.2-1.2 \%$ of all colorectal malignancies (4). The most common variety of colonic lymphoma is non-Hodgkin's 
lymphoma (NHL) (5). Therapeutic strategies for NHL include radical tumor resection plus multiagent chemotherapy for earlystage patients, and biopsy plus multidrug chemotherapy for advanced stage patients.

Due to the low incidence of PCL, most prior studies of this disease were conducted with small sample populations and at a single institution (6-8). Contemporary populationbased studies of PCL have revealed clinical and demographic patient factors that may be associated with the prognosis of colorectal lymphomas $(9,10)$. Chouhan et al. (9) focused on all gastrointestinal tract lymphomas, while Cai et al. (10) limited their study to factors that were associated with prognosis after the surgical resection of PCL. In the current study, we investigated the epidemiological changes of colorectal lymphoma from 1973 to 2014 by analyzing data from the Surveillance, Epidemiology, and End Results (SEER) database. We also reported the incidence by lymphoma subtype and trends in the use of initial surgical therapy for these patients.

\section{METHODS}

\section{Data Collection}

The SEER Cancer Statistics Review (http://seer.cancer.gov/data/ citation.html) is a report on the most recent cancer incidence, mortality, survival, prevalence, and lifetime risk statistics and is published annually by the Data Analysis and Interpretation Branch of the National Cancer Institute, USA. Two cohorts in the SEER database were used in the present study. The cohort of the SEER 9 registry from 1973 to 2014 was used to estimate the long-term incidence of systematic lymphoma and PCL, as previously described (11). A second cohort, the SEER 18 registry, which covers $28 \%$ of the US population, was created to estimate the incidence by histologic subtype, the use of surgical therapy, and survival. To ensure sufficient follow-up time, patients diagnosed after 2012 were excluded from the study.

We extracted all data from cases of lymphoma and lymphoma subtypes from the SEER database. Patients had to have a pathological diagnosis of a lymphoid neoplasm for inclusion. The site of the primary tumor was the colon (including cecum, appendix, right colon, hepatic flexure, transverse colon, splenic flexure, left colon, and sigmoid colon) and the rectum (overlapping lesions and rectum). For PCL, the site codes representing "colon" (C18.0-C18.9); "rectosigmoid junction" (C19.9), and "rectum" (C20.9) according to the Third Edition of International Classification of Diseases for Oncology (ICD-O-3) were used to identify eligible patients. The inclusion criteria in the current study were as follows: (1) Anatomic site of the primary tumor localized in the colon or rectum (ICDO-3: C18.0-18.9, C19.9, and C20.9); (2) Histological type limited to lymphoma (ICD-O-3: 9590-9738); and (3) Malignant behavior (ICD-O-3 code: 3 ). The following subgroup types

\footnotetext{
Abbreviations: CRC, colorectal cancer; SEER, the Surveillance, Epidemiology, and End Results; PCL, primary colorectal lymphoma; NHL, non-Hodgkin's lymphoma; DLBCL, Diffuse large B-cell lymphoma; MZL, marginal zone lymphoma; MALT, mucosa-associated lymphoid tissue; CHOP, cyclophosphamide, doxorubicin, vincristine and prednisone; FDA, Food and Drug Administration; CSS, causespecific survival.
}

were included in this analysis because these types constitute the vast majority of cases: mantle cell lymphoma (ICD-O-3: 9673), diffuse large B-cell lymphoma (9680), Burkitt lymphoma (9687), follicular lymphoma (9690-9698), and marginal zone B-cell lymphoma (9699). The exclusion criteria were: (1) Patients without histological confirmation; and (2) Patients with unclear information regarding surgery. The lymphoma had to be the only primary or the first of multiple primaries.

We acquired permission to access the research data file in the SEER program through the National Cancer Institute, USA; the reference number was 10359-Nov2016. This study was approved by the ethics committee/institutional review board at Fudan University Shanghai Cancer Center.

\section{Statistical Analyses}

Age-adjusted incidence was estimated as diagnoses per $1,000,000$ patients per year (SEER*Stat software, version 8.3.21, Information Management Services, Inc., Calverton, MD). Changes in incidence were assessed and fit (Joinpoint Regression Program version 4.2, Information Management Services, Inc., Calverton, MD) using log-linear models and APCs estimated for the final, best-fitting model. Differences in trends were also assessed $(11,12)$.

Survival rates were generated using Kaplan-Meier curves, and the differences were compared with the log-rank test. Patients who were alive or dead due to other diseases at the last followup were excluded from analysis. The statistical evaluation was conducted with SPSS 22.0 (SPSS Inc., Chicago, IL, USA). All CIs are stated at the $95 \%$ confidence level. Statistical significance was defined as $P<0.05$ (two-sided).

\section{RESULTS}

\section{PCL Incidence}

The PCL incidence increased from 1.4 per 1,000,000 people (hereafter, all incidence estimates are per 1,000,000 people) in 1973 to 3.5 in 2014, with an annual percentage change (APC) of $1.98 \%$ (95\% confidence interval [CI]: $1.29-2.68 \%, P<0.001$ ) from 1985 to 2014 and no statistically significant change between 1973 and 1985 (Figure 1A, blue line). We then investigated whether there were differences in incidence between older and younger people according to a cutoff of age 60. For people younger than 60 years, there was a slight increase in PCL incidence from 0.6 to $1.4 \%$ from 1973 to 1989 , with an APC of $6.38 \%$ (95\% CI: $3.45-9.39 \%, P<0.001$ ), but no statistically significant change thereafter (Figure 1A, cyan line). For people 60 years or older, there was a statistically significant increase in PCL incidence from 5.4 to $14.1 \%$ from 1973 to 2014 . The APC was $4.43 \%$ (95\% CI: $3.50-5.36 \%, P<0.001$ ) from 1973 to 2006 , with no statistically significant change thereafter (Figure 1A, yellow line).

The incidence of systemic lymphoma presentations increased from 136.1 to 226.2 with an APC of 3.22\% (95\% CI: 2.91-3.53\%, $P<0.001$ ) from 1977 to $1990,0.68 \%$ (95\% CI: $0.49-0.88 \%$, $P<0.001$ ) from 1990 to 2007 , and $-1.14 \%$ (95\% CI: $-1.81 \%$ to $-0.46 \%, P=0.002$ ) from 2007 to 2014 . There was no statistically significant change from 1973 to 1977 (Figure 1B, blue line). At 
A

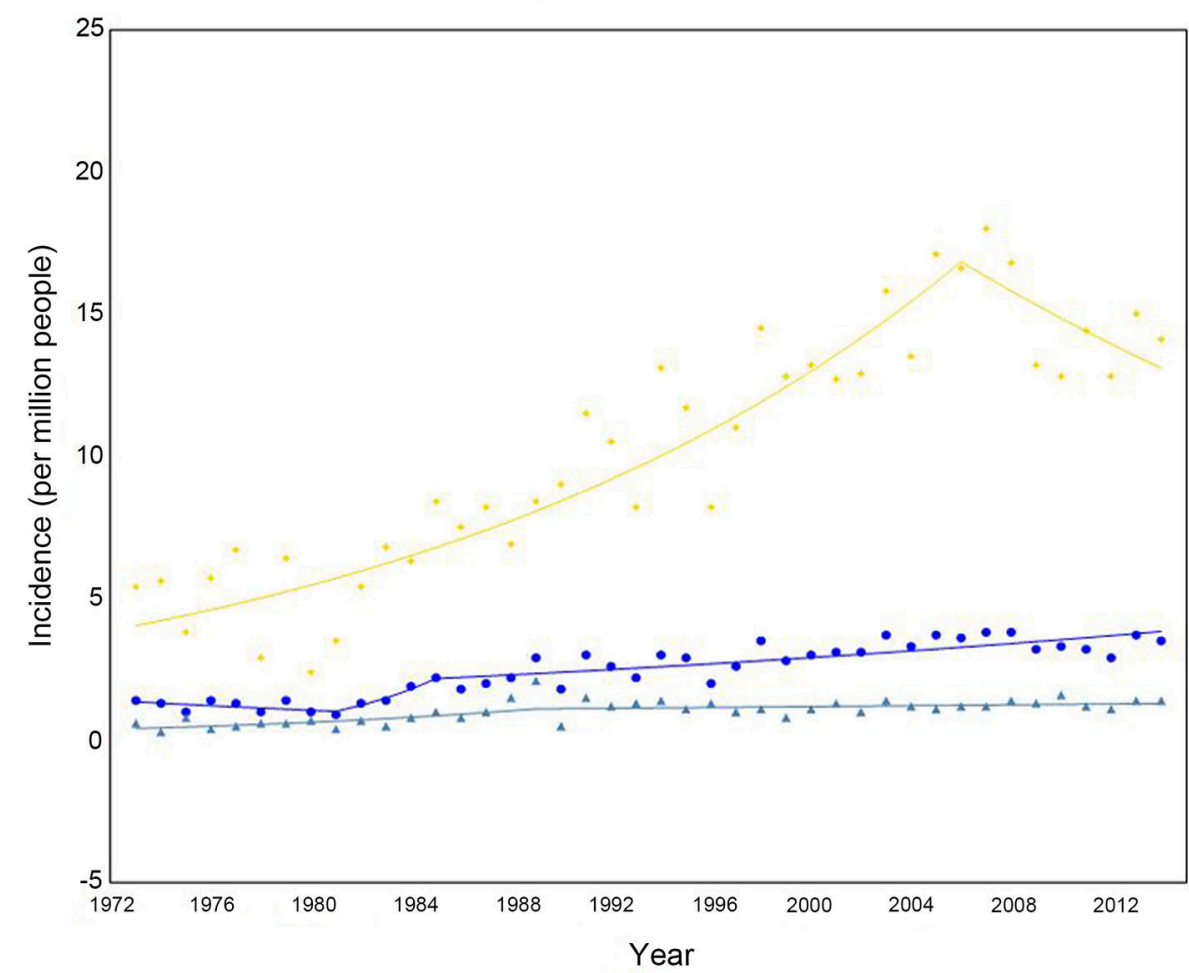

B

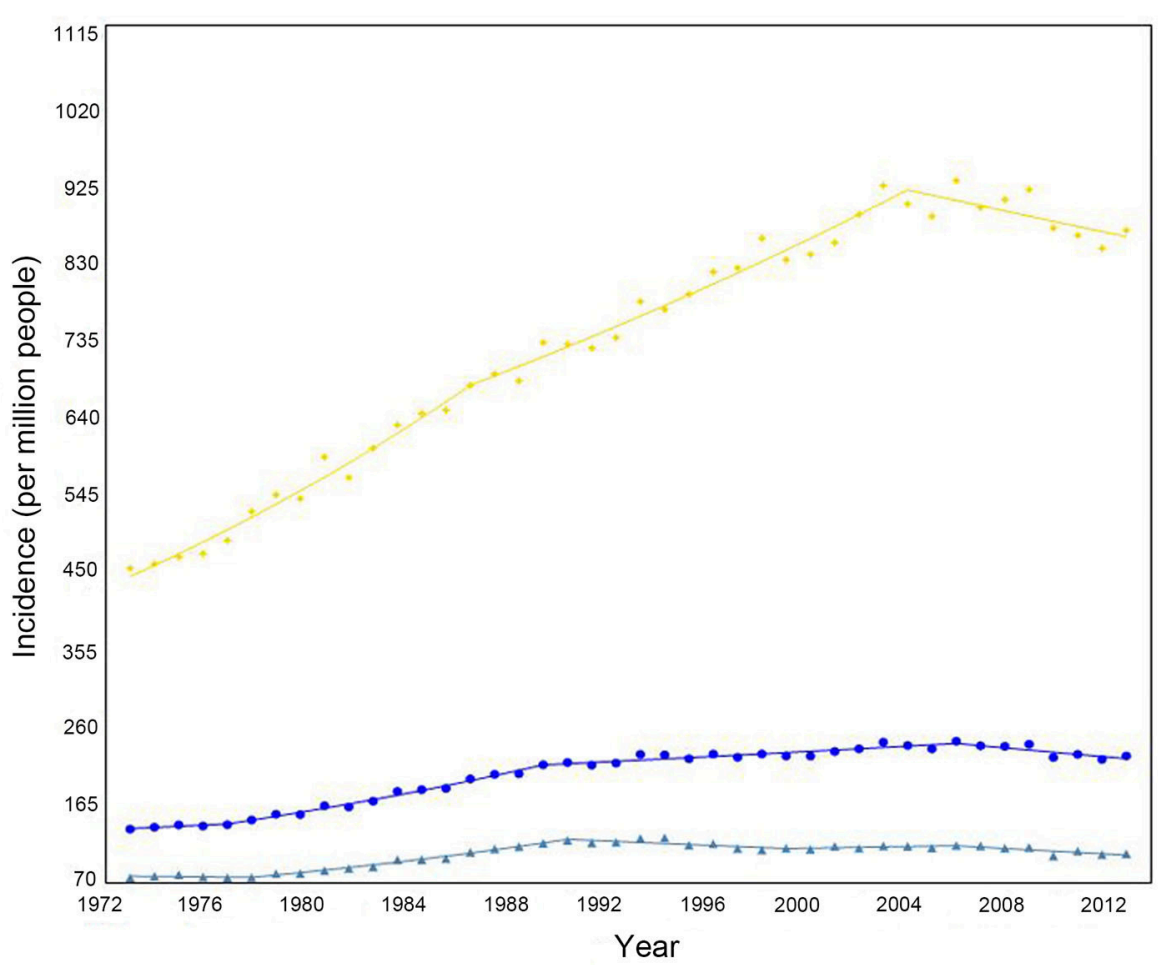

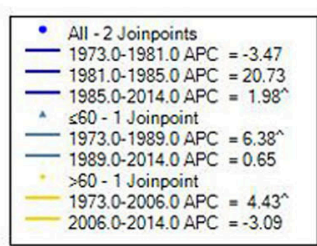

- All - 3 Joinpoints $\begin{aligned} 1973.0-1977.0 \mathrm{APC} & =1.03 \\ -1977.0-1990.0 \mathrm{APC} & =3.22\end{aligned}$ $\begin{aligned} 1990.0-2007.0 \mathrm{APC} & =0.68\end{aligned}$ 2007.0-2014.0 APC $=-1.14^{\prime}$ $\leq 60-4$ Joinpoints

1973.0-1978.0 APC $=-0.38$

1978.0-1991.0 APC $=3.74^{\wedge}$

$1991.0-2000.0 \mathrm{APC}=-1.06$

$2000.0-2007.0$ APC $=0.42$

$2007.0-2014.0$ APC $=-1.46$

$>60-2$ Joinpoints

1973.0-1987.0 APC $=3.07^{\circ}$

1987.0-2005.0 APC $=1.69^{\circ}$

2005.0-2014.0 APC $=-0.71^{\circ}$

FIGURE 1 | Incidence of primary colorectal lymphoma (A) and total lymphoma (B) by age. Incidence (per 1000000 persons) was calculated in SEER 9 registries, 1973-2014. 
same periods, for patients younger than 60 , the APC for systemic lymphoma was 3.75\% (95\% CI: 3.30-4.19\%, $P<0.001$ ) from 1978 to $1991,-1.06 \%$ (95\% CI: -1.84 to $-0.27 \%, P=0.01$ ) from 1991 to 2000 , and $-1.46 \%$ ( $95 \% \mathrm{CI}:-2.41$ to $-0.50 \%, P=0.004)$ from 2007 to 2014. There was no significant change from 1973 to 1978 or from 2000 to 2007 (Figure 1B, cyan line). For patients 60 years or older, the incidence increased from 1973 to 1987 (APC 3.07\%, 95\% CI: $2.77-3.77 \%, P<0.001$ ) and from 1987 to 2005 (APC 1.69\%, 95\% CI: $1.47-1.92 \%, P<0.001)$, but it decreased from 2005 to 2014 (APC $-0.71 \%, 95 \%$ CI: -1.28 to $-0.14 \%, P=0.010$ )

(Figure 1B, yellow line).

\section{PCL Incidence and Presentation by Subtype}

A total of 3,860 eligible patients diagnosed with PCL between 1973 and 2012 were identified in the SEER 18 registry (Table 1). The majority of PCL cases were non-Hodgkin's lymphoma (99.2\%), and the highest proportion was Diffuse large B-cell lymphoma-PCL (DLBCL-PCL) (43.4\%), followed by extranodal marginal zone lymphoma (MZL) (14.2\%) and follicular lymphoma (8.2\%). Notably, the ratio of DLBCL in PCL increased gradually from 1973 to 1977 , peaking at $49.6 \%$ in the period of $1985-1988$ and decreasing to $38.3 \%$ in the period of 2009-2012. Conversely, the proportion of follicular lymphoma increased from $4.1 \%$ in $1973-1976$ to $10.9 \%$ in 2009-2012. The unifying category of "extranodal MZL of mucosa-associated lymphoid tissue (MALT lymphoma)" was proposed more than two decades ago $(13,14)$ and was first recorded in 1995; it accounted for $20.1 \%$ of PCLs in the 20092012 period (Figure 2A).

The most common primary site was the cecum $(34.2 \%)$, followed by the rectum (15.4\%). The median ages at diagnosis were 63, 65, and 63 years for DLBCL-PCL, MZL-PCL and follicular lymphoma, respectively. By comparison, the median age at diagnosis of MZL-PCL was significantly older than that for the other subtypes $(P<0.001$, Mann-Whitney $U$ test $)$. Presentation with stage I disease occurred more frequently in MZL-PCL (67.09\%) than in DLBCL-PCL (36.06\%), follicular lymphoma (48.58\%), and the other subtypes (35.66\%) $(P<0.001$, $\chi^{2}$ test). Specifically, there appeared to be no significant changes in the percentages of different tumor stages from 1983 to 2012 (Figure 2B). Because the Ann Arbor staging system was used only from 1983 onward, patients diagnosed before 1983 were not included in the stage analysis.

\section{PCL Initial Local Therapy}

The proportion of surgical resection changed markedly from 1973 to 2012 (Figure 3). From 1978 to 1980, 83.3-100\% of affected patients underwent surgery. After 1980, the proportion of patients receiving surgery gradually decreased. From 2010 to 2012, the proportion of patients who received local resection at diagnosis decreased to $47.7-52.6 \%$. Conversely, the proportion of patients receiving non-surgical therapy increased gradually; this proportion first exceeded the surgical resection rate in 2008 (Figure 3).
TABLE 1 | Patient and tumor characteristics of primary colorectal lymphoma diagnosed in SEER 18 registries, 1973-2012.

\begin{tabular}{|c|c|c|}
\hline Characteristic & No. & Percentage \\
\hline Sample size & 3860 & 100 \\
\hline Median age, year & 62 & \\
\hline \multicolumn{3}{|l|}{ Age at diagnosis } \\
\hline Younger than age 60 & 1612 & 41.8 \\
\hline Age60 years older & 2248 & 58.2 \\
\hline \multicolumn{3}{|l|}{ Histology } \\
\hline Non-Hodgkin lymphoma & 3831 & 99.2 \\
\hline \multicolumn{3}{|l|}{ B-cell lymphoma } \\
\hline Diffuse large B-cell lymphoma & 1675 & 43.4 \\
\hline Follicular lymphoma & 317 & 8.2 \\
\hline Marginal zone lymphoma & 550 & 14.2 \\
\hline Mantle-cell lymphoma & 281 & 7.4 \\
\hline Burkitt lymphoma & 241 & 6.2 \\
\hline Others & 767 & 20.02 \\
\hline Hodgkin lymphoma & 29 & 0.8 \\
\hline \multicolumn{3}{|l|}{ Stage (Ann Arbor Stage,1983+) } \\
\hline । & 1597 & 41.4 \\
\hline$\|$ & 841 & 21.8 \\
\hline III & 187 & 4.8 \\
\hline IV & 826 & 21.4 \\
\hline Unknown* & 409 & 10.6 \\
\hline \multicolumn{3}{|l|}{ Primary site } \\
\hline Cecum & 1322 & 34.2 \\
\hline Ascending colon & 391 & 10.1 \\
\hline Hepatic flexure & 73 & 1.9 \\
\hline Transverse & 177 & 4.6 \\
\hline Splenic flexure & 49 & 1.3 \\
\hline Descending & 123 & 3.2 \\
\hline Sigmoid colon & 385 & 10.0 \\
\hline Overlapping lesion & 118 & 3.1 \\
\hline Rectosigmoid junction & 104 & 2.7 \\
\hline Colon,NOS & 390 & 10.1 \\
\hline Rectum & 594 & 15.4 \\
\hline
\end{tabular}

*Unknown included 187 cases diagnosed before 1983 and was not staged according to Ann Arbor Stage.

\section{PCL 5-Year Cause-Specific Survival}

The 5-year cause-specific survival (CSS) for PCL improved markedly from 1973 to 2012. For cases diagnosed between 1973 and 1976, the 5-year CSS was 41.6\%, increasing to $80.2 \%$ for cases diagnosed between 2009 and 2012. Improved survival was also seen for systemic lymphoma patients, increasing from 54.6 to $75.0 \%$ over this time period (Figure 4A). There was no apparent difference in CSS between PCL and systemic lymphoma.

Patients who received surgical resection of PCL generally had better CSS than those who did not receive surgical resection (Figure 4B). However, the superiority of surgical resection was significantly reduced after the 1997-2000 time period. We next divided patients into two subgroups-those who underwent surgical resection before or after 2000. In the period of 


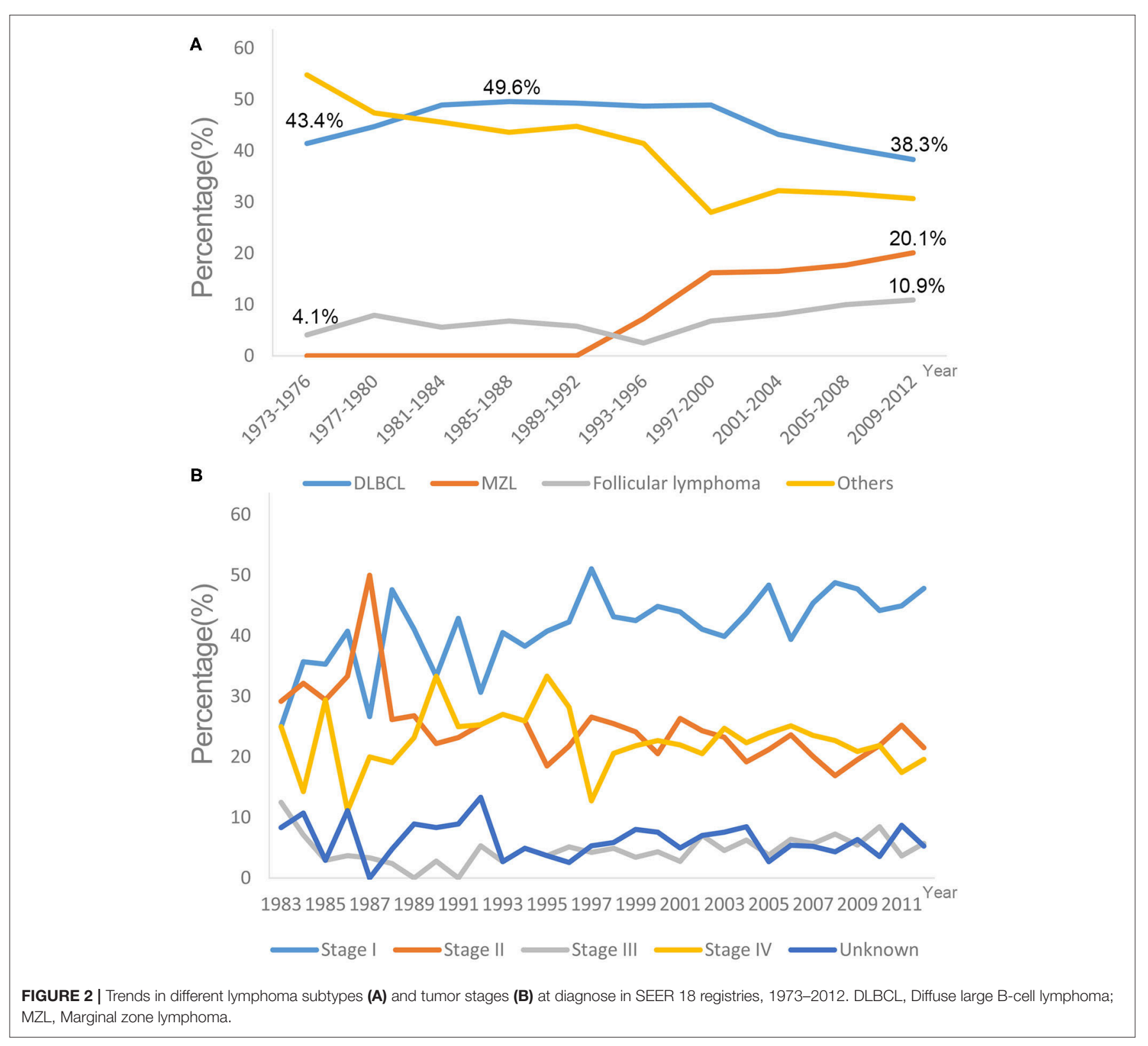

1973-2000, there was an absolute $11.7 \%$ improvement in the 5 -year CSS for patients treated with surgical resection compared with those who did not undergo surgical resection (59.7 vs. $\left.48.0 \%, \chi^{2}=15.268, P<0.001\right)$. For the period of 2001-2012, although the survival difference was still statistically significant, there was only a $4.1 \%$ improvement in 5 -year CSS in patients who received surgical resection $\left(75.9\right.$ vs. $71.8 \%, \chi^{2}=5.373$, $P=0.020)$ (Figure 4C).

\section{DISCUSSION}

Lymphoma involving the colon and rectum is rare and can occur either as primary colorectal lymphoma or as a manifestation of metastasis (2). Owing to its low incidence rate, PCL has not been well-described. To our knowledge, this is the largest contemporary study describing the incidence, initial local therapy, and outcomes of PCL. In our analyses of data spanning 42 years, the overall incidence of PCL increased from 1.4 to 3.5 per $1,000,000$ from 1973 to 2014 . The incidence of PCL in older people was significantly higher than in younger people. The incidence increased from 5.1 to 14.1 per $1,000,000$ elderly people, while it increased from 0.6 to 1.4 per $1,000,000$ young people in the studied time period. The incidence of systemic lymphoma also gradually increased, showing a trend similar to that of PCL.

In the earlier years included in this study, surgical resection was generally recommended to treat PCL in the absence 


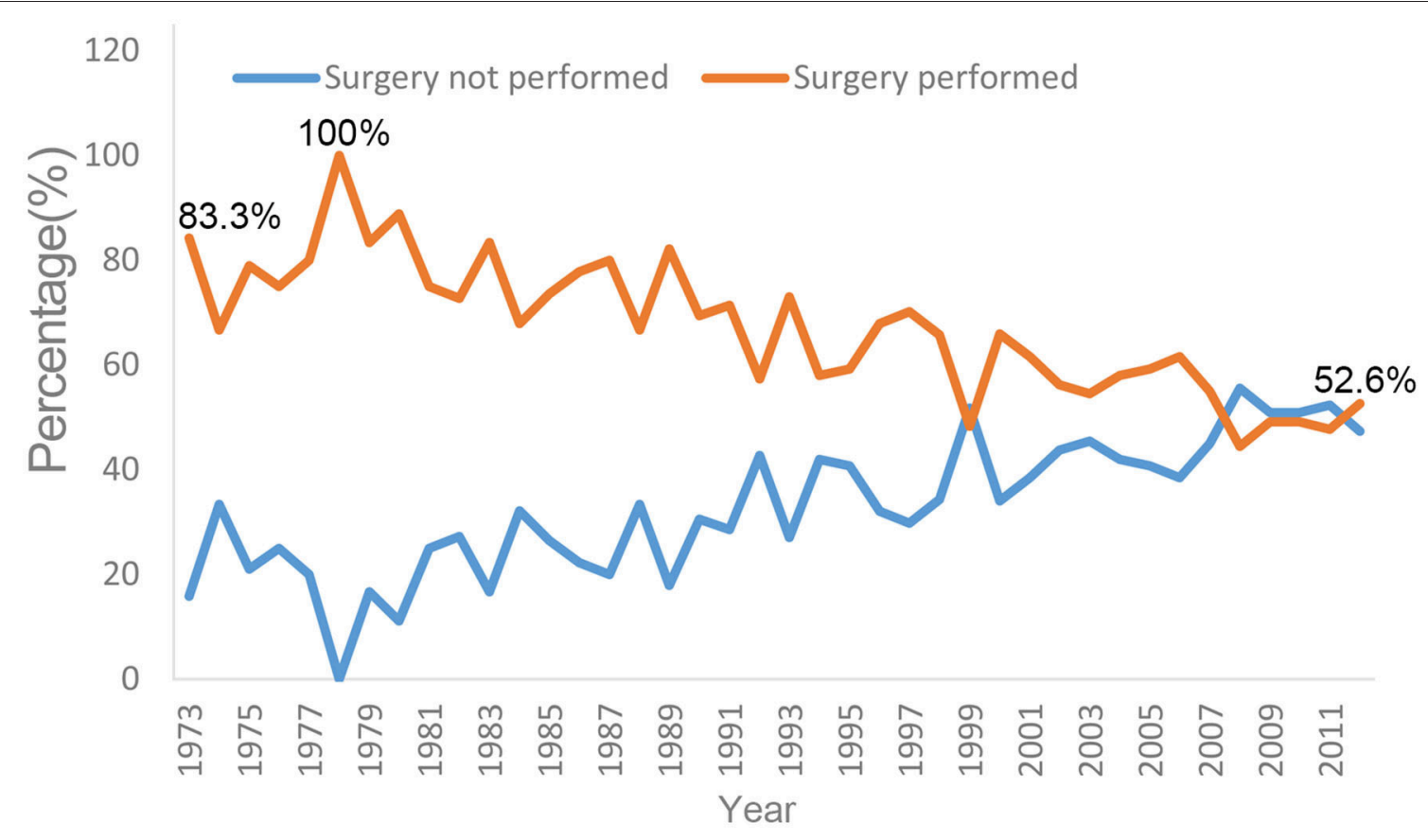

FIGURE 3 | Trends in surgery and non-surgery therapy in SEER 18 registries, 1973-2012.

of metastasis (10, 15-18). However, based on pathological examinations of these patients, only $14-24 \%$ had no regional lymph node metastases (stage IE), while the majority of patients (ranging from 62 to $86 \%$ ) had regional lymph node involvement (stage IIE). Moreover, a small number of patients were ultimately found to have diffuse visceral involvement at the time of laparotomy (stage IVE) (2). These findings suggest that PCL is a systemic disease, indicating that surgery may not be the best treatment for PCL. Some authors have stated that it is beneficial to perform colectomy to prevent spontaneous perforation (5), a complication that has a high rate of occurrence. However, others believe that early diagnosis and timely onset of chemotherapy might be adequate treatments for such patients, thus avoiding surgical intervention (19). Indeed, chemotherapy is effective for treating PCL. The $\mathrm{CHOP}$ chemotherapy regimen (cyclophosphamide, doxorubicin, vincristine, and prednisone) is the standard first-line therapy for B-cell lymphomas. Rituximab, which was approved by the Food and Drug Administration (FDA) in 1997, has led to high response rates and good progression-free, diseasefree and overall survival (20-22). In the present study, we found that the proportion of different tumor stages was not changed over the study period, but the 5-year CSS increased steadily. Conversely, the rate of surgical resection decreased significantly over the study period. These results suggest that the improved survival of PCL patients may be primarily caused by the advancement of chemotherapies and biotargeted therapies. CHOP combination chemotherapy has cured $30 \%$ of patients with diffuse large-cell lymphomas (23). Second- and thirdgeneration regimens with predicted 5-year survival rates of
$>55 \%$ have been recently developed (24-26). The addition of rituximab to the $\mathrm{CHOP}$ regimen has significantly increased the complete response rate and has prolonged event-free and overall survival (20-22).

This study was limited by the extent of information available in the SEER database. No information on therapeutic strategies was available, so we cannot directly assess the impact of this increasingly important treatment parameter. Furthermore, we do not know the type of surgery performed. As the majority of PCLs have lymph node metastases, it is unknown whether extensive lymphadenectomy offers a survival benefit. The unifying category of lymphoma was first proposed in $1994(13,14)$, which leads to the objective difficulty in recognizing lymphomas prior to 1994, resulting in the possible underestimation of incidence before this time. Finally, the SEER database lacks information on the education, income status, insurance status, and marriage status of patients, which have been validated as prognostic factors $(27,28)$, and we could not adjust for these factors in the survival analysis.

\section{CONCLUSION}

PCL remains a rare form of extranodal lymphoma. The data presented here demonstrated the increasing incidence of PCL throughout the study period. Concurrently, the PCL survival outcomes increased continuously over this time period, while the surgical resection rate decreased steadily. Changes in the survival trends and therapy 


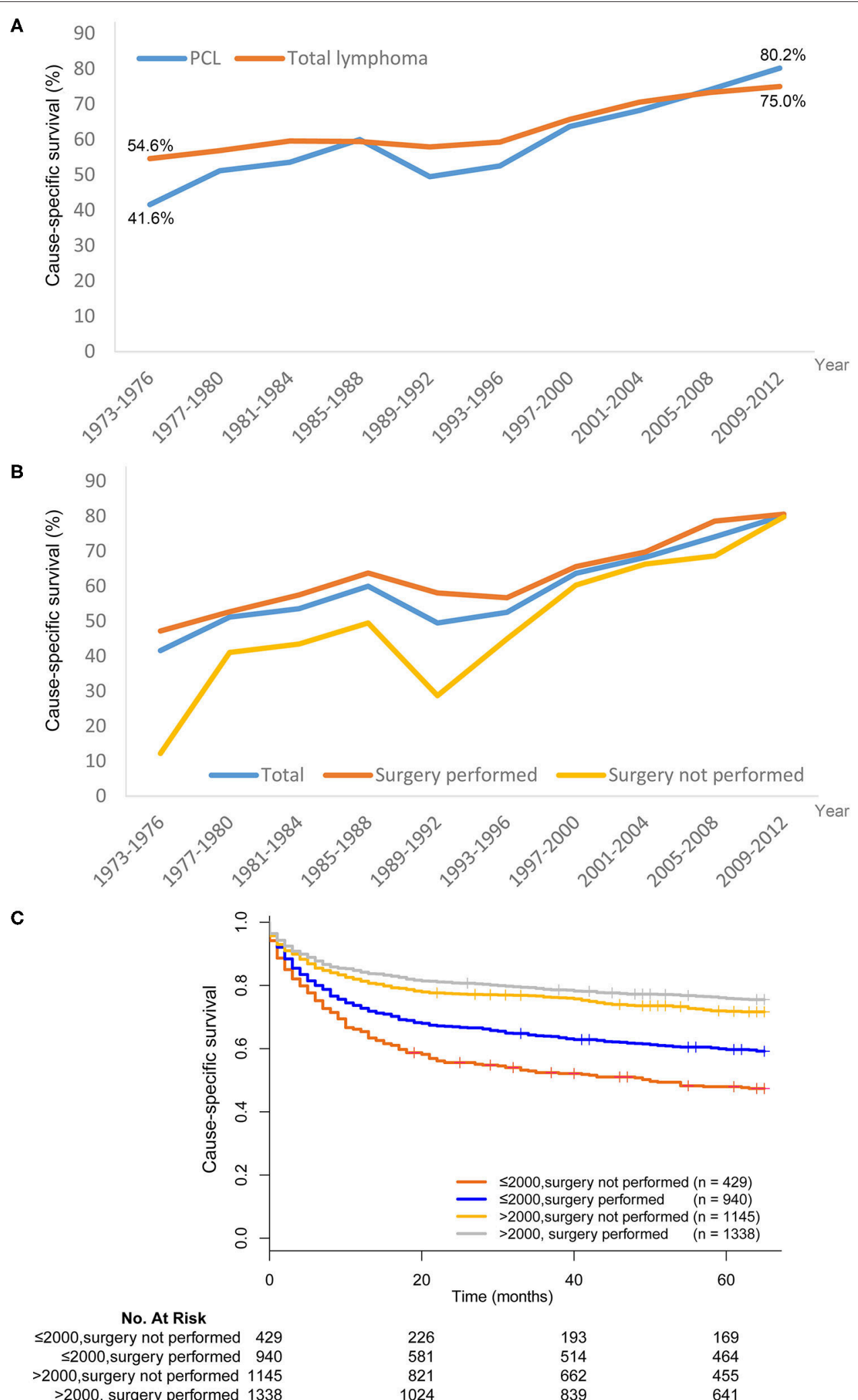

FIGURE 4 | Trends in cause-specific survival (CSS) of lymphoma in SEER 18 registries, 1973-2012. (A) The CSS was improved in both primary colorectal lymphoma (PCL) and total lymphoma. (B) Patients who received surgical resection of PCL generally had better CSS than those without surgical resection. (C) The superiority of surgical resection was significantly reduced after year 1997-2000. 
strategies indicate that PCL can be well-managed with newer therapeutic reagents. Additional investigations should be carried out to further verify the interesting findings presented here.

\section{DATA AVAILABILITY STATEMENT}

All datasets generated for this study are included in the article/Supplementary Material.

\section{ETHICS STATEMENT}

Ethical approval was not provided for this study on human participants because this study was completely based on the publicly available SEER database and we have got the permission to access them on purpose of research only. It did not include interaction with humans or use personal identifying information. The informed consent was not required for this research. Written informed consent for participation was not required for this study in accordance with the national legislation and the institutional requirements.

\section{REFERENCES}

1. Siegel RL, Miller KD, Jemal A. Cancer Statistics, 2017. CA Cancer J Clin. (2017) 67:7-30. doi: 10.3322/caac. 21387

2. Quayle FJ, Lowney JK. Colorectal lymphoma. Clin Colon Rectal Surg. (2006) 19:49-53. doi: 10.1055/s-2006-942344

3. Zucca E, Roggero E, Bertoni F, Cavalli F. Primary extranodal nonHodgkin's lymphomas. Part 1: gastrointestinal, cutaneous and genitourinary lymphomas. Ann Oncol. (1997) 8:727-37. doi: 10.1023/A:1008282818705

4. Wong MT, Eu KW. Primary colorectal lymphomas. Colorectal Dis. (2006) 8:586-91. doi: 10.1111/j.1463-1318.2006.01021.x

5. Bairey O, Ruchlemer R, Shpilberg O. Non-Hodgkin's lymphomas of the colon. Isr Med Assoc J. (2006) 8:832-5.

6. Nam MJ, Kim BC, Park SC, Hong CW, Han KS, Sohn DK, et al. Mucosa-associated lymphoid-tissue lymphoma of the cecum and rectum: a case report. Ann Coloproctol. (2017) 33:35-38. doi: 10.3393/ac.2017.33.1.35

7. Eso Y, Nishikawa Y, Takai A. Follicular lymphoma of the caecum resected endoscopically. Arab J Gastroenterol. (2016) 17:143-4. doi: 10.1016/j.ajg.2016.08.003

8. Katsumata R, Matsumoto H, Motoyasu O, Murao T, Ishii M, Fujita M, et al. Primary colorectal lymphoma comprising both components of diffuse large B-cell lymphoma and mucosa-associated lymphoid tissue lymphoma combined with cytomegalovirus colitis. Clin J Gastroenterol. (2016) 9:59-62. doi: 10.1007/s12328-016-0642-y

9. Chouhan J, Batra S, Gupta R, Guha S. Gastrointestinal follicular lymphoma: using primary site as a predictor of survival. Cancer Med. (2016) 5:2669-77. doi: $10.1002 / \mathrm{cam} 4.763$

10. Cai YB, Chen HY, He JJ, Hu YT, Yang Q, Chen LB, et al. The role of surgical intervention in primary colorectal lymphoma: a SEER population-based analysis. Oncotarget. (2016) 7:72263-75. doi: 10.18632/oncotarget.12344

11. Thomas A, Link BK, Altekruse S, Romitti PA, Schroeder MC. Primary breast lymphoma in the United States: 1975-2013. J Natl Cancer Inst. (2017) 109:djw294. doi: 10.1093/jnci/djw294

12. Kim HJ, Fay MP, Yu B, Barrett MJ, Feuer EJ. Comparability of segmented line regression models. Biometrics. (2004) 60:1005-14. doi: 10.1111/j.0006-341X.2004.00256.x

13. Schmid C, Kirkham N, Diss T, Isaacson PG. Splenic marginal zone cell lymphoma. Am J Surg Pathol. (1992) 16:455-66. doi: 10.1097/00000478-199205000-00004

\section{AUTHOR CONTRIBUTIONS}

QL, SM, and WD planned the study. SM and WD calculated statistics and analyzed the data. YL and QL wrote the manuscript. $\mathrm{XL}$, SC, and GC supervised the entire project. All authors reviewed the manuscript.

\section{FUNDING}

This study was partially supported by grants from the National Science Foundation of China (Nos. 81702353, 81372646, 81101586, and 81472222), Shanghai Municipal Natural Science Foundation (17ZR1406400). The funders had no role in the study design, data collection and analysis, decision to publish, or preparation of the manuscript.

\section{ACKNOWLEDGMENTS}

The authors acknowledge the efforts of the Surveillance, Epidemiology, and End Results (SEER) Program tumor registries in the creation of the SEER database. The interpretation and reporting of these data are the sole responsibility of the authors.

14. Isaacson PG. Gastric MALT lymphoma: from concept to cure. Ann Oncol. (1999) 10:637-45. doi: 10.1023/A:1008396618983

15. Doolabh N, Anthony T, Simmang C, Bieligk S, Lee E, Huber P, et al. Primary colonic lymphoma. J Surg Oncol. (2000) 74:257-62. doi: 10.1002/ 1096-9098(200008)74:4<257::AID-JSO3>3.0.CO;2-U

16. Zighelboim J, Larson MV. Primary colonic lymphoma. Clinical presentation, histopathologic features, and outcome with combination chemotherapy. J Clin Gastroenterol. (1994) 18:291-7. doi: 10.1097/00004836-199406000-00007

17. Contreary K, Nance FC, Becker WF. Primary lymphoma of the gastrointestinal tract. Ann Surg. (1980) 191:593-8. doi: 10.1097/00000658-198005000-00011

18. Aviles A, Neri N, Huerta-Guzman J. Large bowel lymphoma: an analysis of prognostic factors and therapy in 53 patients. J Surg Oncol. (2002) 80:111-5. doi: $10.1002 /$ jso. 10103

19. Stanojevic GZ, Nestorovic MD, Brankovic BR, Stojanovic MP, Jovanovic MM, Radojkovic MD. Primary colorectal lymphoma: an overview. World J Gastrointest Oncol. (2011) 3:14-8. doi: 10.4251/wjgo.v3.i1.14

20. Morrison VA. Evolution of R-CHOP therapy for older patients with diffuse large B-cell lymphoma. Exp Rev Anticancer Ther. (2008) 8:1651-8. doi: 10.1586/14737140.8.10.1651

21. Subramanian J, Cavenagh J, Desai B, Jacobs I. Rituximab in the treatment of follicular lymphoma: the future of biosimilars in the evolving therapeutic landscape. Cancer Manag Res. (2017) 9:131-40. doi: 10.2147/CMAR.S120589

22. Coiffier B, Lepage E, Briere J, Herbrecht R, Tilly H, Bouabdallah R, et al. CHOP chemotherapy plus rituximab compared with CHOP alone in elderly patients with diffuse large-B-cell lymphoma. N Engl J Med. (2002) 346:235-42. doi: 10.1056/NEJMoa011795

23. Nicolaides C, Dimou S, Pavlidisa N. Prognostic factors in aggressive non-hodgkin's lymphomas. Oncologist. (1998) 3:189-97. doi: 10.1634/theoncologist.3-3-189

24. Shipp MA, Yeap BY, Harrington DP, Klatt MM, Pinkus GS, Jochelson MS, et al. The m-BACOD combination chemotherapy regimen in large-cell lymphoma: analysis of the completed trial and comparison with the M-BACOD regimen. J Clin Oncol. (1990) 8:84-93. doi: 10.1200/JCO.1990.8.1.84

25. Longo DL, DeVita VT, Duffey PL, Wesley MN, Ihde DC, Hubbard SM, et al. Superiority of ProMACE-CytaBOM over ProMACE-MOPP in the treatment of advanced diffuse aggressive lymphoma: results of a prospective randomized trial. J Clin Oncol. (1991) 9:25-38. doi: 10.1200/JCO.1991.9.1.25 
26. Coiffier B, Gisselbrecht C, Herbrecht R, Tilly H, Bosly A, Brousse N. LNH-84 regimen: a multicenter study of intensive chemotherapy in 737 patients with aggressive malignant lymphoma. J Clin Oncol. (1989) 7:1018-26. doi: 10.1200/JCO.1989.7.8.1018

27. Li Q, Gan L, Liang L, Li X, Cai S. The influence of marital status on stage at diagnosis and survival of patients with colorectal cancer. Oncotarget. (2015) 6:7339-47. doi: 10.18632/oncotarget.3129

28. Pulte D, Jansen L, Brenner H. Social disparities in survival after diagnosis with colorectal cancer: contribution of race and insurance status. Cancer Epidemiol. (2017) 48:41-7. doi: 10.1016/j.canep.2017. 03.004
Conflict of Interest: The authors declare that the research was conducted in the absence of any commercial or financial relationships that could be construed as a potential conflict of interest.

Copyright (C) $2020 \mathrm{Li}, \mathrm{Mo}$, Dai, Li, Xu, Li, Cai and Cai. This is an open-access article distributed under the terms of the Creative Commons Attribution License (CC BY). The use, distribution or reproduction in other forums is permitted, provided the original author(s) and the copyright owner(s) are credited and that the original publication in this journal is cited, in accordance with accepted academic practice. No use, distribution or reproduction is permitted which does not comply with these terms. 